können mittels Ultraschall gut diagnostiziert werden, so Koch. Bei Tumoren im tiefen Drüsenlappen oder parapharyngealen Tumoren ist ein MRT erforderlich. Finden sich Malignitätszeichen, erfolgen ein MRT und ein CT mit Kontrastmittel. Eine PET-CT ist nicht sinnvoll, um Speicheldrüsenkarzinome zu diagnostizieren, ist jedoch für das pri-

\title{
... Therapie von Speicheldrüsenerkankungen
}

n der Therapie von inflammatorischen und obstruktiven Speicheldrüsenerkrankungen sei der Drüsenerhalt heute Standard, berichtete Koch. Primär kommen konservative Maßnahmen wie antiinflammatorische Medikamente, Antibiotika oder Drüsen-Massagen mit Sialogoga zum Einsatz. Spezifische Infektionen, System-Erkrankungen oder Autoimmunerkrankungen bedürfen einer interdisziplinären Betreuung.

Bei eindeutigen Zeichen einer Obstruktion ist die interventionelle Sialendoskopie Methode der Wahl, um die Ursache zu beheben. Gute Erfolge lassen sich damit auch bei der chronisch-rezidivierenden juvenilen Parotitis erzielen, wie eine aktuelle Metaanalyse bestätigt [Ramakrishna J et al.: Laryngoscope 2015; 125 (6): 1472-1479].

Speichelsteine können meist gut endoskopisch entfernt werden, so Koch. Eine neuere Entwicklung bei größeren oder ungünstig gelegenen Steinen sei die intraduktale Lithotripsie mittels Laser oder pneumatischer Fragmentierung [Koch $\mathrm{M}$ et al.: Laryngoscope 2016; 126 (7): 1545-1550]. Weitere wichtige Therapiemöglichkeiten sind die extrakorporale Stoßwellenlithotripsie (ESWL) und die transorale Gangchirurgie. Bei therapieresistenten Steinen in der Glandula parotis ist die Kombination von Sialendoskopie und transkutaner Chirurgie ein weiteres erfolgversprechendes Verfahren.

Eine Kortison-Instillation erfolgt insbesondere bei Sialodochitis und Stenosen. Auch bei Stenosen stellt die interventionelle Sialendoskopie die Therapie der ersten Wahl dar. Gegebenenfalls kann ein Stent implantiert werden. Wird eine Ablation der Drüsenfunkti- märe Staging und die Therapieplanung bei High-grade-Malignomen sowie für die Detektion von Fernmetastasen wertvoll. Die Grobnadel-Biopsie sollte bei Speicheldrüsentumoren gegenüber der Feinnadel-Punktion bevorzugt werden, sagte Koch. In vielen Fällen sei jedoch erst die offene Biopsie wegweisend.

\section{Hier steht eine Anzeige.}

on notwendig, favorisiert Koch die Injektion von Botulinum-Toxin. Die operative Entfernung der Drüse ist die Ultima ratio, aber nur in wenigen Fällen notwendig.

Benigne Tumoren der Glandula submandibularis werden durch Entfernung der gesamten Drüse therapiert. Bei benignen Tumoren der Glandula parotis zeichne sich dagegen ein Paradigmenwechsel ab, so Koch. Hier wird heute ein Drüsenerhalt angestrebt. Interessante Entwicklungen der letzten Jahre seien drüsenerhaltende Operationstechniken wie die partielle superfizielle Parotidektomie und insbesondere die extrakapsuläre Dissektion [Xie S et al.: Medicine (Baltimore) 2015; 94 (34): e1237].

Malignome werden mittels kompletter und gegebenenfalls erweiterter Drüsenentfernung therapiert, wenn notwendig mit Resektion der Halslymphknoten und in Kombination mit einer adjuvanten Therapie. Bei der Therapie von Low-grade-Malignomen der Glandula parotis mit Primärtumorstadium T1-2 könnte aber eine limitierte Parotidektomie eine Option für die Zukunft sein, weitere Studien bleiben abzuwarten, so Koch.

Koch M.: Überblick über Diagnostik und Therapie von Speicheldrüsenerkrankungen

Berichterstattung: Angelika Bauer-Delto 